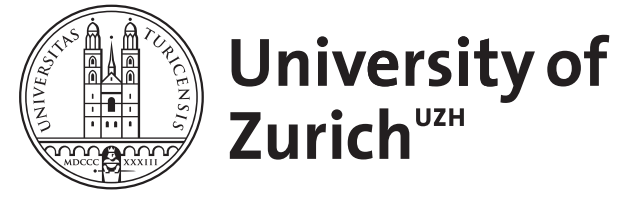

\title{
Topology of the Type IIa Na+/Pi Cotransporter
}

\author{
Radanovic, T ; Gisler, S M ; Biber, J ; Murer, H
}

\begin{abstract}
The type IIa $\mathrm{Na}(+) / \mathrm{P}(\mathrm{i})$ cotransporter (NaPi-IIa) plays a key role in the reabsorption of inorganic phosphate $(\mathrm{P}(\mathrm{i}))$ in the renal proximal tubule. The rat NaPi-IIa isoform is a protein of 637 residues for which different algorithms predict 8-12 transmembrane domains (TMDs). Epitope tagging experiments demonstrated that both the $\mathrm{N}$ and the $\mathrm{C}$ termini of NaPi-IIa are located intracellularly. Sitedirected mutagenesis revealed two N-glycosylation sites in a large putative extracellular loop. Results from structure-function studies suggested the assembly of two similar opposed regions that possibly constitute part of the substrate translocation pathway for one phosphate ion together with three sodium ions. Apart from these topological aspects, other structural features of NaPi-IIa are not known. In this study, we have addressed the topology of NaPi-IIa using in vitro transcription/translation of HK-M0 and HK-M1 fusion vectors designed to test membrane insertion properties of cDNA sequences encoding putative NaPi-IIa TMDs. Based on the results of in vitro transcription/translation analyses, we propose a model of NaPiIIa comprising 12 TMDs, with both $\mathrm{N}$ and $\mathrm{C}$ termini orientated intracellularly and a large hydrophilic extracellular loop between the fifth and sixth TMDs. The proposed model is in good agreement with the prediction of the NaPi-IIa structure obtained by the hidden Markov algorithm HMMTOP.
\end{abstract}

DOI: https://doi.org/10.1007/s00232-006-0033-2

Posted at the Zurich Open Repository and Archive, University of Zurich

ZORA URL: https://doi.org/10.5167/uzh-2745

Journal Article

Published Version

Originally published at:

Radanovic, T; Gisler, S M; Biber, J; Murer, H (2006). Topology of the Type IIa Na+/Pi Cotransporter. Journal of Membrane Biology, 212(1):41-49.

DOI: https://doi.org/10.1007/s00232-006-0033-2 


\title{
Topology of the Type IIa Na ${ }^{+} / \mathrm{P}_{\mathrm{i}}$ Cotransporter
}

\author{
Tamara Radanovic, Serge M. Gisler, Jürg Biber, Heini Murer \\ Institute of Physiology and Center for Integrative Human Physiology, University of Zurich, Winterthurerstrasse 190, CH-8057-Zurich, \\ Switzerland
}

Received: 18 May 2006/Revised: 6 September 2006

\begin{abstract}
The type IIa Na ${ }^{+} / \mathrm{P}_{\mathrm{i}}$ cotransporter (NaPiIIa) plays a key role in the reabsorption of inorganic phosphate $\left(\mathrm{P}_{\mathrm{i}}\right)$ in the renal proximal tubule. The rat $\mathrm{NaPi}$-IIa isoform is a protein of 637 residues for which different algorithms predict 8-12 transmembrane domains (TMDs). Epitope tagging experiments demonstrated that both the $\mathrm{N}$ and the $\mathrm{C}$ termini of $\mathrm{NaPi}$-IIa are located intracellularly. Site-directed mutagenesis revealed two $\mathrm{N}$-glycosylation sites in a large putative extracellular loop. Results from structure-function studies suggested the assembly of two similar opposed regions that possibly constitute part of the substrate translocation pathway for one phosphate ion together with three sodium ions. Apart from these topological aspects, other structural features of NaPi-IIa are not known. In this study, we have addressed the topology of NaPi-IIa using in vitro transcription/translation of $\mathrm{HK}-\mathrm{M} 0$ and $\mathrm{HK}$ M1 fusion vectors designed to test membrane insertion properties of cDNA sequences encoding putative NaPi-IIa TMDs. Based on the results of in vitro transcription/translation analyses, we propose a model of NaPi-IIa comprising 12 TMDs, with both $\mathrm{N}$ and $\mathrm{C}$ termini orientated intracellularly and a large hydrophilic extracellular loop between the fifth and sixth TMDs. The proposed model is in good agreement with the prediction of the NaPi-IIa structure obtained by the hidden Markov algorithm HMMTOP.
\end{abstract}

Key words: $\mathrm{Na}^{+} / \mathrm{P}_{\mathrm{i}}$ cotransporter $-\mathrm{NaPi}$-IIa Secondary structure - HK-M0 - HK-M1 - In vitro transcription/translation

Correspondence to: Jürg Biber; email: JuergBiber@access.unizh.ch

\section{Introduction}

In renal proximal tubules and small intestine, transepithelial transport of inorganic phosphate $\left(\mathrm{P}_{\mathrm{i}}\right)$ is mediated by type II $\mathrm{Na}^{+} / \mathrm{P}_{\mathrm{i}}$ cotransporters, members of the solute carrier family SLC34 (Murer, Forster \& Biber, 2004). This transporter family comprises three members, the type IIa (SLC34A1), the type IIb (SLC34A2) and the type IIc (SLC34A3) $\mathrm{Na}^{+} / \mathrm{P}_{\mathrm{i}}$ cotransporters. These cotransporters are localized at the apical membranes of proximal tubular and small intestinal epithelial cells. Transport of $\mathrm{P}_{\mathrm{i}}$ is strictly dependent on the presence of sodium ions, yet the stoichiometry $\left(\mathrm{Na}^{+}\right.$ions $/ \mathrm{P}_{\mathrm{i}}$ ions) differs. Both types IIa and IIb Na ${ }^{+} / P_{i}$ cotransporters transport three $\mathrm{Na}^{+}$ions together with one $\mathrm{HPO}_{4}{ }^{2-}$ ion and thus operate in an electrogenic manner. On the other hand, the type IIc isoform operates in an electroneutral manner with a stoichiometry of 2:1 (Bacconi et al., 2005; Segawa et al., 2002).

On the basis of several predictions, all SLC34 family members share a common overall secondary structure that most likely comprises eight transmembrane domains (TMDs), and both termini are located intracellularly (Forster et al., 2002). Based on site-directed mutagenesis followed by electrophysiological analyses, two symmetrical regions, each containing opposed reentrant loops, have been proposed to contribute to the overall transport pathway of $\mathrm{Na}^{+}$and $\mathrm{HPO}_{4}{ }^{2-}$ ions (Kohler et al., 2002a, 2002b).

In this work, we have further investigated the topology of the type IIa $\mathrm{Na}^{+} / \mathrm{P}_{\mathrm{i}}$ (NaPi-IIa) cotransporter as a paradigm of the SLC34 family by taking advantage of the in vitro translation HK-M0/HK-M1 assay. This system is composed of two vectors encoding fusion proteins of 101 (HK-M0) or 139 (HK-M1) amino acids derived from the $\beta$-subunit of $\mathrm{H}^{+} / \mathrm{K}^{+}$-ATPase with a linker region and 177 amino acids derived from the $\beta$-subunit of $\mathrm{H}^{+} / \mathrm{K}^{+}$-ATPase. The presence of a TMD in the HK-M0 linker region 
would translocate the reporter sequence into the lumen of microsomes and therefore cause $\mathrm{N}$-glycosylation of the fusion protein; such a sequence is defined as a signal anchor (SA) sequence. On the other hand, a TMD positioned in the HK-M1 vector would translocate the reporter sequence out of the lumen of microsomes, thus preventing $N$-glycosylation; such an amino acid sequence has been characterized as a stop transfer (ST) sequence (Zizak et al., 2000).

This tool has successfully been applied to study the topology of numerous solute transporters, e.g., the gastric $\mathrm{H}^{+} / \mathrm{K}^{+}$-ATPase (Bamberg \& Sachs, 1994), the endoplasmic reticulum $\mathrm{Ca}^{2+}$-ATPase (Bayle, Weeks \& Sachs, 1995), the human ileal $\mathrm{Na}^{+}$dependent bile acid cotransporter HISBAT (Hallen et al., 1999, 2002), a P-type ATPase from Heliobacter pylori (Melchers et al., 1996), the rabbit gastric cholecystokinin-A (CCK-A) receptor (Bayle, Weeks \& Sachs, 1997b), the $\mathrm{Na}^{+} / \mathrm{K}^{+} / 2 \mathrm{Cl}^{-}$cotransporter NKCC1 (Gerelsaikhan \& Turner, 2000), the $\mathrm{Na}^{+} /$ $\mathrm{H}^{+}$exchanger NHE-3 (Zizak et al., 2000) and the $\mathrm{Na}^{+}$-bicarbonate cotransporter pNBCI (Tatishchev et al., 2003).

Transmembrane regions in question of the NaPiIIa cotransporter were chosen according to the outcome of the hidden Markov algorithm HMMTOP (Tusnady \& Simon, 2001) because this prediction was in best agreement with our previous experimental findings, notably also pointing to an intracellular orientation of both $\mathrm{N}$ and $\mathrm{C}$ termini (Hayes et al., 1994).

\section{Materials and Methods}

\section{Prediction of Transmembrane Sequences}

Several different computer algorithms were used to predict the secondary structure of the NaPi-IIa protein: Kyte-Doolittle (Kyte \& Doolittle, 1982), SOSUI (Hirokawa, Boon-Chieng \& Mitaku, 1998), TMpred (Hofmann \& Stoffel, 1993), PRED-TMR (Pasquier et al., 1999), ALOM (Klein, Kanehisa \& DeLisi, 1985), MTOP (Hartmann, Rapoport \& Lodish, 1989) and two programs derived from the hidden Markov model, TMHMM (Krogh et al., 2001) and HMMTOP (Tusnady \& Simon, 2001).

\section{Vector Constructions}

Expression plasmids HK-M0 and HK-M1 (kindly provided by Dr. George Sachs, University of California, Los Angeles, CA) were used to analyze the topological properties of TMDs of NaPi-IIa by transcription/translation assays. Originally, these vectors were constructed from the backbone of pGEM7zf $+\Delta H$ indIII (Promega, Madison, WI) and have been described in detail elsewhere (Bamberg \& Sachs, 1994). Sequences coding for putative TMDs of $\mathrm{NaPi}-\mathrm{II} a$ were cloned into the $B g / \mathrm{II} / H i n d \mathrm{III}$ linker region, between the $\alpha$ - and $\beta$-subunits, whose glycosylation status was used to determine the location of the $\mathrm{C}$ terminus of the fusion protein (Bayle et al., 1997b).

\section{Polymerase Chain Reaction and Ligation}

Sequences coding for putative TMDs of NaPi-IIa were synthesized by polymerase chain reaction (PCR, cloned $P f u$ polymerase; Stratagene, La Jolla, CA) using the full-length rat isoform harbored in pSPORT1 (accession number L13257) as a template. Sense primers contained a $B g / I I$ site, while antisense primers contained a HindIII site. PCR products were digested with BglII and HindIII enzymes and ligated into HK-M0 and HK-M1 vectors according to standard protocols. Correct insertion of all PCR products was verified by sequencing (Microsynth, Balgach Switzerland).

\section{IN Vitro Transcription/Translation Assays}

The membrane insertion properties of chosen sequences were determined by transcription/translation assays. The fusion proteins were expressed using the TNT Quick Coupled Transcription/ Translation system (Promega, Madison, WI) in the presence of $\left[{ }^{35} \mathrm{~S}\right]$ L-methionine (Hartmann, Braunschweig Germany) and, if indicated, in the presence of canine pancreatic microsomal membranes (Promega) according to the manufacturer's protocol.

\section{Sodium Dodecyl Sulfate-Polyacrylamide Gel ElECTROPHORESIS AND AUTORADIOGRAPHY}

The translation products were separated on $10 \%$ sodium dodecyl sulfate-polyacrylamide gel electrophoresis (SDS-PAGE) gels. Samples were denatured at $70^{\circ} \mathrm{C}$ for $10 \mathrm{~min}$ in $2 \%$ SDS, $1 \mathrm{~mm}$ ethylenediaminetetraacetic acid (EDTA), $10 \%$ glycerol, $85 \mathrm{~mm}$ Tris/HCl (pH 6.8) and $100 \mathrm{~mm}$ dithiothreitol (DTT). After electrophoresis, gels were fixed for $15 \mathrm{~min}$ in $50 \%$ methanol plus $10 \%$ glacial acetic acid and washed in $10 \%$ acetic acid for 15 min and distilled water for $40 \mathrm{~min}$. After drying at $80^{\circ} \mathrm{C}$, the gels were subjected to autoradiography for $1-3$ days at $-80^{\circ} \mathrm{C}$.

\section{EXPRESSION IN XENOPUS LAEVIS OOCYTES, \\ Surface Biotinylation and Streptavidin Precipitation}

A missense mutation, S183C, was introduced into the rat NaPi-IIa by PCR (Pfu-ultraHF, Stratagene, La Jolla, CA) using the following primers: sense, 5'-GCTTGTTGGAGGTGAGCTGCGCCATTCCGATAATCATGGG, and antisense, 5'-CCCATGATT ATCGGAATGGCGCAGCTCACCTCCAACAAGC. Another mutant used in this study, S460C, has been previously described (Lambert et al., 1999a). cRNAs of wild-type NaPi-IIa and the mutants S460C and S183C were synthesized according to standard protocols (MEGAScript T7 Kit; Ambion, Austin, TX). Oocytes from $X$. laevis were prepared as described (Kohler et al., 2002b), injected with $15 \mathrm{ng}$ of each cRNA and incubated for 3 days at $16^{\circ} \mathrm{C}$. To label exposed cysteine residues of expressed proteins, 15 oocytes per group were incubated with 1 mм MTSEA-biotin $(N$ biotinylaminoethylmethanethiosulfonate; Sigma, St. Louis, MO) in ND100 buffer (100 mм NaCl, 2 mм KCl, $1.8 \mathrm{~mm} \mathrm{CaCl}_{2}, 1 \mathrm{~mm}$ $\mathrm{MgCl}_{2}, 10 \mathrm{~mm}$ 4-(2-hydroxyethyl)-1-piperazineethanesulfonic acid [HEPES]/Tris [pH 7.4]) for $10 \mathrm{~min}$ at room temperature. After washing five times in $1 \mathrm{ml}$ of ND100, the oocytes were homogenized in $300 \mu$ of homogenization buffer (HB; $100 \mathrm{~mm} \mathrm{NaCl}, 1 \mathrm{~mm}$ EDTA, $5 \mu \mathrm{g} / \mathrm{ml}$ leupeptin, $5 \mu \mathrm{g} / \mathrm{ml}$ pepstatin A, $20 \mathrm{~mm}$ Tris/ $\mathrm{HCl}$ [pH 7.6]) by pipetting and passing through a $25 \mathrm{G}$ needle. To remove yolk proteins, the homogenates were centrifuged twice at $200 \times g$ for $5 \mathrm{~min}$ at $4^{\circ} \mathrm{C}$. Proteins were solubilized by diluting supernatants $1: 1$ with $\mathrm{HB}$ containing $1 \%$ sodium deoxycholate by vigorous pipetting followed by vortexing for 20 s. Precipitation 
Table 1. Prediction of transmembrane topology of NaPi-IIa

\begin{tabular}{|c|c|c|c|c|c|c|c|}
\hline & Kyte-Doolittle & SOSUI & TMpred & PRED-TMR & ALOM & ТМНММ & НМMTOP \\
\hline $\mathrm{N}$ terminus & - & - & Extracellular & - & Intracellular & Extracellular & Intracellular \\
\hline $\mathrm{C}$ terminus & - & - & Intracellular & - & Extracellular & Intracellular & Intracellular \\
\hline TMD1 & $102-124$ & $103-125$ & $101-124$ & $106-125$ & $106-122$ & $106-128$ & $106-128$ \\
\hline TMD2 & $145-167$ & $140-162$ & $141-162$ & $141-160$ & $147-163$ & $141-163$ & $141-158$ \\
\hline TMD3 & $172-196$ & $169-191$ & - & $169-189$ & $169-185$ & - & $165-182$ \\
\hline TMD4 & - & - & $187-205$ & - & - & $183-205$ & $187-204$ \\
\hline TMD5 & $225-243$ & $222-244$ & $222-240$ & - & - & $225-247$ & $225-244$ \\
\hline TMD6 & $343-374$ & $347-369$ & $346-368$ & $350-368$ & $350-366$ & $346-368$ & $346-368$ \\
\hline TMD7 & $400-415$ & $406-428$ & $395-416$ & $395-414$ & $398-414$ & $391-413$ & $393-412$ \\
\hline TMD8 & $418-436$ & - & $412-435$ & $417-435$ & $419-435$ & $418-435$ & $417-434$ \\
\hline TMD9 & - & - & $443-461$ & $441-459$ & - & $442-459$ & $441-460$ \\
\hline TMD10 & $474-487$ & $467-489$ & $471-489$ & $469-489$ & $469-485$ & $469-491$ & $471-490$ \\
\hline TMD11 & $514-537$ & $510-532$ & $511-531$ & $511-531$ & $512-528$ & $511-533$ & $511-533$ \\
\hline TMD12 & $542-558$ & $539-561$ & $538-561$ & $538-556$ & $540-556$ & $537-559$ & $538-559$ \\
\hline
\end{tabular}

with streptavidin was performed with $500 \mu \mathrm{l}$ of solubilized oocyte lysate mixed with $900 \mu \mathrm{l}$ of HB containing $0.1 \%$ Triton X-100 and $60 \mu \mathrm{l}$ of prewashed ImmunoPure Immobilized Streptavidin Gel (Pierce, Rockford, IL). After incubation by slow end-over-end rotation for $2 \mathrm{~h}$ at $4^{\circ} \mathrm{C}$, the samples were washed four times with HB containing $0.1 \%$ Triton X-100 and finally once with HB. Proteins were eluted in $40 \mu \mathrm{l}$ of $2 \mathrm{x}$ SDS sample buffer (4\% SDS, $2 \mathrm{~mm}$ EDTA, 20\% glycerol, $200 \mathrm{~mm}$ DTT, $190 \mathrm{~mm}$ Tris/HCl [pH 6.8]) by boiling for $2 \mathrm{~min}$ at $96^{\circ} \mathrm{C}$. Immunoblots were performed as previously described using a rabbit polyclonal antibody raised against a synthetic N-terminal peptide of the rat NaPi-IIa protein (Custer et al., 1994).

\section{${ }^{32} \mathbf{P}_{\mathrm{I}}$ UPTAKe}

The procedure for ${ }^{32} \mathrm{P}_{\mathrm{i}}$ uptake has been described elsewhere (Werner et al., 1990). Uptake was measured 3 days after injection of both water- and cRNA-injected oocytes $(n=8-10$ oocytes per group). Eventually, prior to uptake, oocytes were treated with 1 mм MTSEA for $10 \mathrm{~min}$ in NC100 buffer (see above).

\section{Results}

In this study, we addressed the topology of NaPi-IIa by an in vitro translation approach using the vectors HK-M0 and HK-M1, which have been successfully applied to study the topology of various solute transporters. Various algorithms were first applied to decide which partial amino acid sequence of the $\mathrm{NaPi}$-IIa protein most likely represents a hydrophobic sequence (HS) and thus can be regarded as a putative TMD (see Table 1). These predictions suggested a secondary structure of NaPi-IIa that comprises 9-12 TMDs. Discrepancies between the different predicted structures are situated in two long hydrophobic segments between amino acid residues 165-244 and 412-460. For the region 165-244, the HMMTOP algorithm predicted three TMDs, whereas only two or one TMD were predicted by the other algorithms. Concerning the region 412-460, two TMDs were predicted by HMMTOP, TMHMM,
PRED-TMR and TMpred, whereas one or none was evident by other algorithms. In addition, the orientation of the $\mathrm{N}$ and $\mathrm{C}$ termini was inconsistent (Table 1); only HMMTOP showed both termini facing the cytosol. As such an arrangement of the $\mathrm{N}$ and $\mathrm{C}$ termini was in agreement with our previous work (Lambert et al., 1999b), HSs predicted by HMMTOP were chosen and tested for their membrane insertion properties.

As illustrated in Figure 1 (control), transcription/ translation of the fusion protein encoded by HK-M0 alone did not result in a shift in the apparent molecular weight when the reaction was performed in the presence of microsomes. This indicated that, due to the absence of an HS with membrane insertion properties, the $\beta$-subunit contained within the HKM0 fusion protein was not translocated into the lumen of the microsomes and therefore was not $\mathrm{N}$-glycosylated. In contrast, the product obtained from the HK-M1 vector showed a shift of $12.5 \mathrm{kDa}$ when the reaction was performed in the presence of microsomes due to $N$-glycosylation of the $\beta$-subunit. This indicated the existence of an HS, namely the first TMD of the $\alpha$-subunit of the $\mathrm{H}^{+} / \mathrm{K}^{+}$-ATPase, which was capable of acting as an SA sequence (Bamberg \& Sachs, 1994). For reasons not known, the fusion protein contained in HK-M1 was only partially $N$-glycosylated. Nevertheless, the signal intensity, as illustrated in Figure 1 (control), was always taken as a reference.

First, the hydrophobic sequences HS1, HS2, HS6, HS7, HS11 and HS12 (see Table 2) that were predicted by HMMTOP to be likely TMD 1, 2, 6, 7, 11 and 12 were tested (Table 1). After insertion of HS 1, 2, 6 and 11 in HK-M0, $N$-glycosylation of the reporter $\beta$-subunit in the presence of microsomes was detected (Fig. 1), suggesting that these amino acid sequences acted as SA sequences. After insertion of these HS sequences into HK-M1, no $N$-glycosylations were 


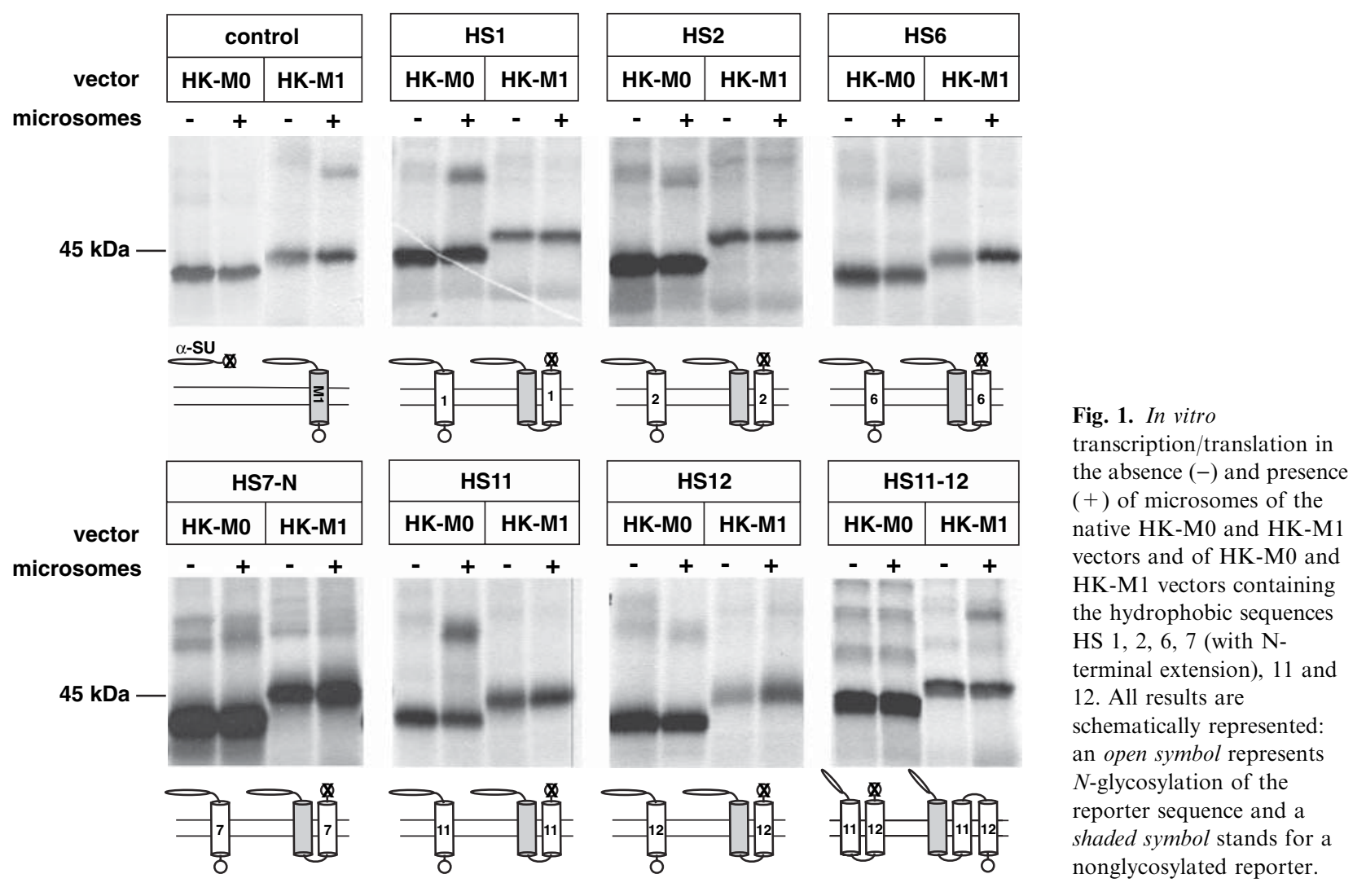

Table 2. Hydrophobic sequences of NaPi-IIa inserted in HK-M0 and HK-M1 vectors and summary of in vitro translation results

\begin{tabular}{|c|c|c|c|c|c|}
\hline \multirow[b]{2}{*}{ Insert } & \multirow[b]{2}{*}{ Tested sequence } & \multirow[b]{2}{*}{ Position } & \multicolumn{2}{|c|}{ Glycosylation } & \multirow[b]{2}{*}{ Activity } \\
\hline & & & HK-M0 & HK-M1 & \\
\hline HS1 & LLKVPL—FQLAGG & $101-130$ & + & - & $\mathrm{SA} / \mathrm{ST}$ \\
\hline HS2 & DNAILS-VLVQSS & $139-163$ & + & - & $\mathrm{SA} / \mathrm{ST}$ \\
\hline HS3 & SSSTST—LEVSSA & $162-184$ & - & + & $-1-$ \\
\hline HS4 & IPIIMG-QAGDRT & $185-211$ & - & + & $-1-$ \\
\hline HS3-4 & SSSTST - QAGDRT & $162-211$ & - & + & \\
\hline HS5 & AFAGAT-LHHVTG & $219-249$ & - & + & $-/-$ \\
\hline HS6 & LPDLAV_ILLVKM & $343-370$ & + & - & $\mathrm{SA} / \mathrm{ST}$ \\
\hline HS7 & DFPAPF-SMTFVV & $389-414$ & - & - & $-/ \mathrm{ST}$ \\
\hline HS7-N & KGQVAN_SMTFVV & $376-414$ & + & - & $\mathrm{SA} / \mathrm{ST}$ \\
\hline HS8 & QSSSVF-VISIER & $415-437$ & - & + & $-/-$ \\
\hline HS9 & RAYPLT_EKLSSS & $437-468$ & - & + & $-1-$ \\
\hline HS8-9 & QSSSVF-EKLSSS & $415-468$ & - & + & \\
\hline HS10 & FQIALC - YPLPCT & $469-492$ & - & + & $-/-$ \\
\hline HS11 & YRWFAV_FGISMA & $509-534$ & + & - & $\mathrm{SA} / \mathrm{ST}$ \\
\hline HS12 & QAMVGV-NVLQSR & $537-562$ & + & - & $\mathrm{SA} / \mathrm{ST}$ \\
\hline HS11-12 & YRWFAV-NVLQSR & $509-562$ & - & + & \\
\hline
\end{tabular}

observed, which was in agreement with their ST properties.

No clear SA property for HS12 was observed when inserted alone. However, insertion of a tandem construct, HS11-HS12, into HK-M1 clearly resulted in an $\mathrm{N}$-glycosylated product, suggesting a configuration as schematically illustrated (Fig. 1).
Amino acids at positions 389-414, predicted by HMMTOP to be TMD 7 (Table 2), did not show membrane integration properties (data not shown). However, when this sequence was N-terminally extended to amino acids 376 (Table 2), $N$-glycosylation of the fusion protein was promoted when inserted in HK-M0 showing SA properties (Fig. 1). The 


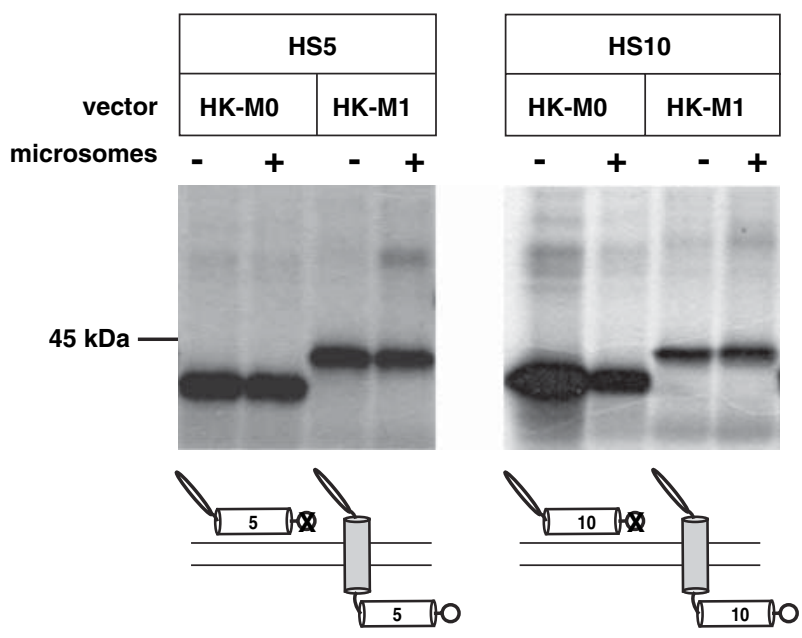

Fig. 2. SDS-PAGE analysis of $\left[{ }^{35} \mathrm{~S}\right]$ Met-labeled fusion proteins obtained by in vitro transcription/translation of the HK-M0 and HK-M1 vectors containing the putative hydrophobic sequences HS5 and HS10 in the absence (-) and presence (+) of microsomes.

same sequence, when inserted into HK-M1, did not cause glycosylation of the translated product and therefore exhibited ST ability.

Even though HS5 and HS10 were predicted to be putative TMDs, results from transcription/translation analysis were not conclusive (Fig. 2).

Taken together, HS 1, 2, 6, 7, 11 and 12 exhibited clear SA or ST properties and, therefore, most likely represent TMDs of NaPi-IIa, whereas no clear results were obtained for the predicted TMDs 5 and 10.

As mentioned, predictions for TMDs 3, 4, 8 and 9 varied among the different algorithms. Single insertions of the respective hydrophobic sequences HS 3, 4, 8 and 9 in HK-M0 and HK-M1 vectors did not provide evidence for SA or ST properties of these sequences (Fig. 3).

Next, HS3 in tandem with HS4 and HS8 in tandem with HS9 showed $N$-glycosylation after transcription/translation of HK-M1 (Fig. 3). As illustrated in Figure 3, these observations can be interpreted in two ways: (1) HS3 and HS8 may act as SA sequences, while HS4 and HS9 may act as ST sequences; or (2) there may be no SA or ST properties of these hydrophobic sequences.

In an earlier study, we demonstrated that position 460 within the region HS 8,9 and 10 is oriented toward the external medium (Lambert et al., 1999a). In a similar approach, the mutant $\mathrm{S} 183 \mathrm{C}$ was constructed and analyzed for MTSEA accessibility from the outer membrane surface. First, ${ }^{32} \mathrm{P}_{\mathrm{i}}$ uptake measurements were performed after expression of the wild-type NaPi-IIa or the S183C mutant in X. laevis oocytes (Fig. 4A). Both the wild-type and mutated $\mathrm{NaPi}$-IIa proteins exhibited the same transport rates. In agreement with earlier studies (Lambert et al., 1999a), MTSEA treatment did not affect $\mathrm{Na}^{+} / \mathrm{P}_{\mathrm{i}}$ cotransport of the wild-type protein, while $\mathrm{Na}^{+} / \mathrm{P}_{\mathrm{i}}$ cotransport mediated by the S183C mutant was reduced by approximately half after MTSEA treatment, indicating that the cysteine residue at position 183 was accessible from the outer membrane surface.

To confirm this conclusion, streptavidin precipitation was performed (Fig. 4B). Consistent with the above transport data, after MTSEA treatment, the S183C mutant was precipitated but the wild-type NaPi-IIa protein was not. As a positive control, another mutant, S460C, was included (Lambert et al., 1999a) to validate the labeling procedure. Thus, these experiments indicated that the amino acid 183, which is positioned between HS3 and HS4, must be exposed to the outer membrane surface.

\section{Discussion}

So far, the structure of the NaPi-IIa cotransporter has been modeled by combining predictions of hydropathy algorithms and accessibility information obtained after applying the substituted cysteine accessibility method (SCAM). The model derived from these approaches entailed eight putative TMDs and two reentrant membrane loops covering amino acid residues $\sim 170-204$ and $\sim 425-460$, respectively. Furthermore, evidence was obtained that both termini of NaPi-IIa are located intracellularly and that Asn-298 as well as Asn-328 are N-glycosylated within a large extracellular loop spanning amino acids 250-340 (Forster et al., 2002). In this study, we have systematically tested the membrane insertion properties of putative TMDs from the NaPi-IIa protein that were predicted by the HMMTOP algorithm. A similar strategy was effectively applied to determine membrane topologies of several different integral membrane proteins (Bamberg \& Sachs, 1994; Bayle et al., 1995, 1997a, 1997b; Gerelsaikhan \& Turner, 2000; Hallen et al., 1999, 2002; Mareninova et al., 2005; Tatishchev et al., 2003; Zizak et al., 2000). To clarify our findings, we also used site-directed mutants to test accessibility by membraneimpermeable thiol reagents. Based on the reported findings, the putative TMDs of NaPi-IIa were divided into three groups.

First, in agreement with predictions from all hydropathy algorithms (see Table 1), the hydrophobic sequences representing the putative TMD 1, 2, 6, 7 (with N-terminal extension), 11 and 12 exhibited membrane integration properties when inserted into the vectors HK-M0 and HK-M1. Therefore, these sequences were assigned as a backbone of the secondary structure of the NaPi-IIa protein (Fig. 5, shadowed TMDs).

A second category is represented by the hydrophobic sequences HS 3, 4, 8 and 9. When inserted alone, these HSs did not show any SA or ST 

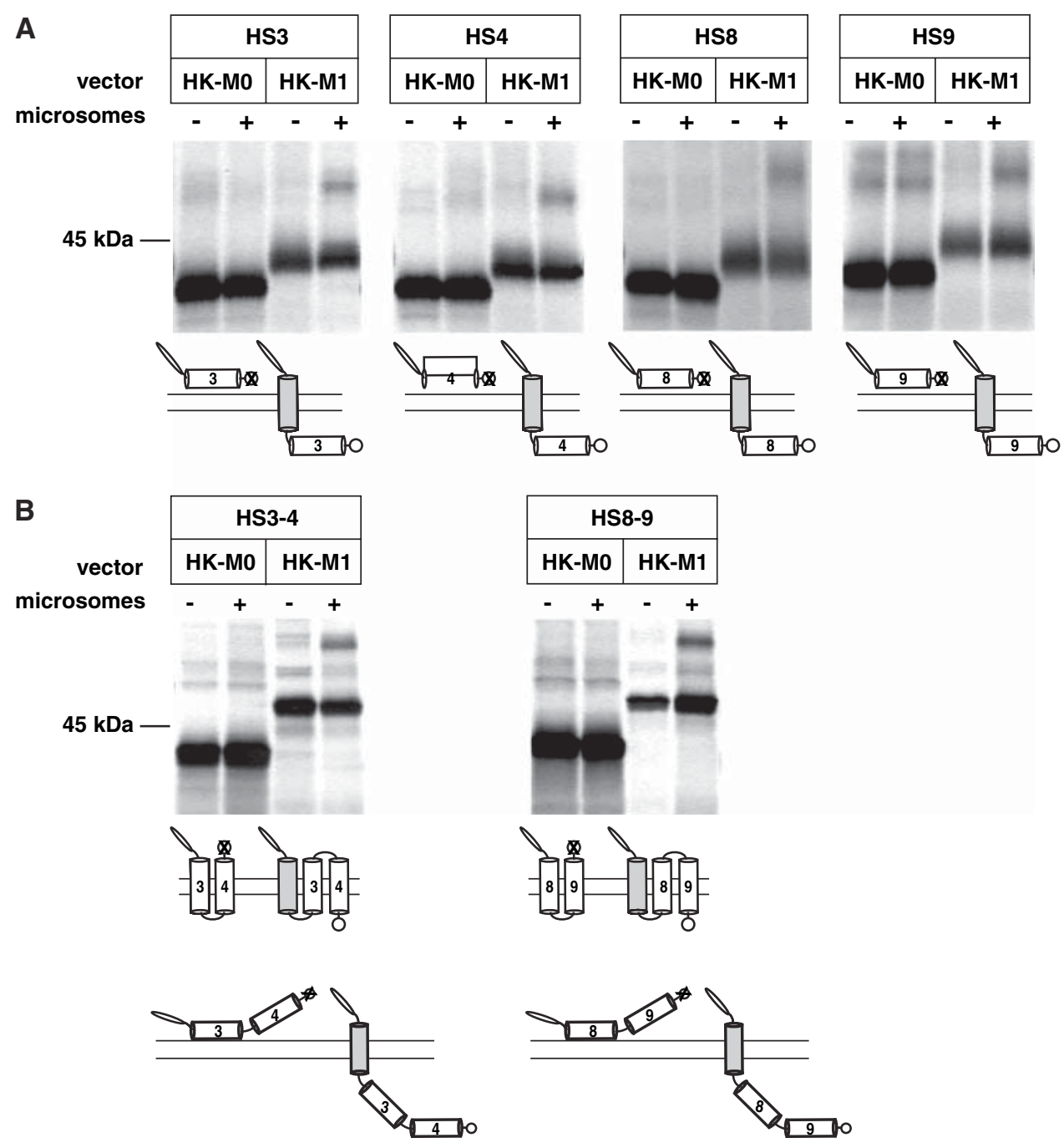

Fig. 3. In vitro transcription/translation of the HK-M0 and HK-M1 vectors with insertions corresponding to putative hydrophobic sequences HS3, HS4, HS8, HS9 ( $A$ ) and tandem insertions of HS3-4 and HS8-9 (B). Two different interpretations are illustrated schematically.

properties. In this respect, it is of interest that major discrepancies in prediction were obtained from these segments (Table 1) despite having lengths large enough to span the membrane. To determine the membrane intruding propensities of these HSs, the tandem constructs HS3-4 and HS8-9 were tested and the results were interpreted in two ways: HS3-4 and HS8-9 may be inserted into the membrane as a pair of TMDs or may be not inserted at all. Monne, Hermansson \& von Heijne (1999) found that some amino acids, such as Pro, Asn, Arg, Lys, His, Glu, Asp and Gln, can cause a turn propensity when placed in the middle of a 40-residue hydrophobic poly(Leu) transmembrane helix, thus converting this segment from a single helix to a pair of helices. Consistent with this hypothesis, we note that Pro-186 is located in the middle of HS3-4, whereas Glu-436 and Arg-437 are close to the middle of HS8-9, which suggests that HS3-4 and HS8-9 may be composed of two helices. A similar conclusion was drawn for TMD9-10 and TMD11-12 of the NKCC1 transporter (Gerelsaikhan \& Turner, 2000).
To confine the bend region within the HS3-4 region, a Cys-mutant S183C located between HS3 and HS4 was used for accessibility experiments with the membrane-impermeable thiol reagent MTSEA. After expression of this mutant in $X$. laevis oocytes, $\mathrm{P}_{\mathrm{i}}$-transport measurements and streptavidin precipitations provided convincing evidence that the site Ser-183 is exposed and accessible from the extracellular medium. Interestingly, the hypothesis of a membrane-inserted HS3-4 pair is further supported from studies using the flounder NaPi-IIb isoform, where the site Ser- 155 that corresponds to Ser-183 of the rat NaPi-IIa could be labeled with a membraneimpermeable fluorophore from the outside (Virkki, Murer \& Forster, 2006a).

The residue Ser-460 is located at the very end of the predicted TMD9 (Lambert et al., 1999a). Since we have shown membrane integration of TMD7 and TMD11, Ser-460 can be positioned either at the very top of the membrane bilayer (Fig. 5A) or at its bottom (Fig. 5B). However, MTSEA-biotinylation and extensive functional studies in the presence of MTS 

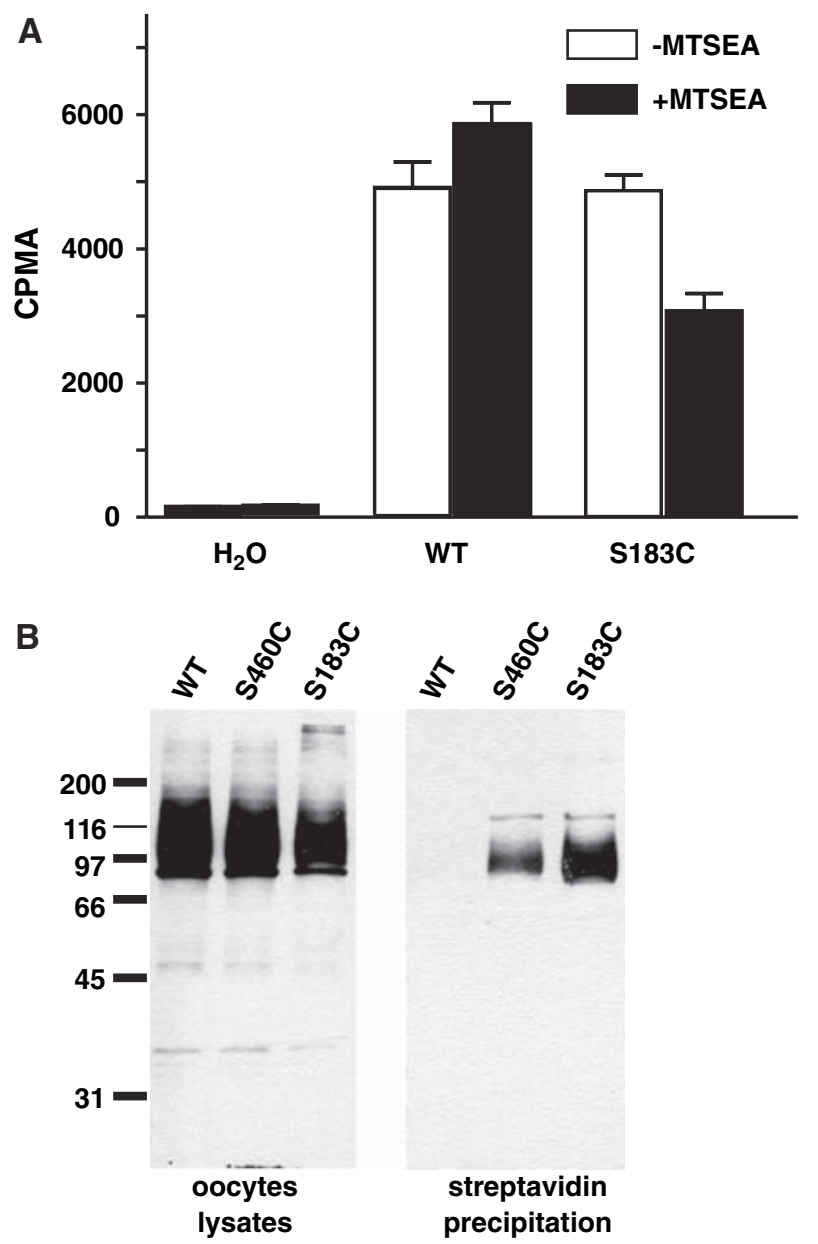

Fig. 4. Effect of the membrane-impermeable thiol reagent MTSEA on wild-type (WT) and S183C-mutated NaPi-IIa after expression in $X$. laevis oocytes. $(A) \mathrm{Na}^{+}$-dependent ${ }^{32} \mathrm{P}_{\mathrm{i}}$-uptake in $X$. laevis oocytes injected with water or wild-type or S183C-mutated NaPiIIa RNA. Uptakes were performed with untreated oocytes or oocytes incubated with $1 \mathrm{~mm}$ MTSEA. Bars represent means \pm standard deviation ( $n=8-10$ oocytes). (B) Western blots of lysates and of streptavidin precipitates obtained after surface MTSEA-biotinylation using oocytes expressing NaPi-IIa and the mutants S460C and S183C.

reagents $\left({ }^{32} \mathrm{P}_{\mathrm{i}}\right.$ uptake and electrophysiology) revealed that the Cys-mutant of Ser-460 is only accessible from the extracellular medium (Lambert et al., 1999a, 1999b). Furthermore, the Cys-mutant S448C of flounder $\mathrm{NaPi}-\mathrm{IIb}$, which corresponds to that of rat S460C, can be labeled with the membrane-impermeable fluorescence dye MTS-rhodamine, and the degree of labeling was shown to depend on the conformational state of the protein (Virkki, Murer \& Forster, 2006). Again, this confirms the exposure of this site to the extracellular environment. When combined with results from in vitro translations and the symmetrical order of TMDs, we can infer that HS8 and HS9, similar to HS3 and HS4, probably stand for a putative pair of TM8-9 from NaPi-IIa.
Thirdly, the group comprising HS5 and HS10 did not insert into the membrane, although they were predicted by most algorithms to be putative TMDs. Interestingly, similar observations have been reported using the same method for various known transmembrane segments such as TM5 +6 of the $\mathrm{H}^{+} / \mathrm{K}^{+}$ATPase and $\mathrm{Ca}^{2+}$-ATPase (Bamberg \& Sachs, 1994; Bayle et al., 1995), $\mathrm{H} 4+8$ of the liver $\mathrm{Na}^{+}$-dependent bile acid transporter (Mareninova et al., 2005) and $\mathrm{H} 4+10$ of pNBC1 (Tatishchev et al., 2003).

A priori, the failure of HS 5 and 10 to insert into the membrane would point to a domain where TMD3-4 (or TMD8-9) is merged to constitute one single and kinked TMD followed by a soluble and external TMD5 (or TMD10), as depicted in Figure 5B. Nevertheless, we propose that both HS3-5 and HS8-10 are positioned within the membrane (Fig. 5A). In HS5, MTSEA labeling of consecutive Cys-mutants at sites Val-222 to Thr-241 revealed that only Ala-240 was accessible from the outside (Virkki et al., 2005). Therefore, an external and nontransmembranous HS5, as indicated in Figure 5B, is unlikely. Similarly, strict cysteine accessibility at position of Ser-460 from the outside (Lambert et al., 1999a; Virkki et al., 2006) suggests that HS10, like HS5, is most probably not situated outside of the membrane, as shown in Figure 5B.

In conclusion, we propose a model of the $\mathrm{NaPi}$-IIa protein containing 12 TMDs with $\mathrm{N}$ and $\mathrm{C}$ termini located intracellularly and a large extracellular loop that spans from $\sim 250$ to $\sim 340$ amino acids and contains two $N$-glycosylation sites. Only the HMMTOP algorithm based on the hidden Markov model predicted a topology of NaPi-IIa that fully complied with experimental observations from our group in recent years (Kohler et al., 2002a, 2002b, 2003; Lambert et al., 1999a, 1999b, 2000, 2001). Clearly, an in vitro transcription/translation analysis may only partially reflect the intracellular environment and, thus, must be interpreted with caution when defining actual membrane domains of a multisegment integral membrane protein. Therefore, our current results need to be confirmed by other methods.

The authors thank Ian C. Forster for critical comments on the manuscript. This work was financially supported by the Swiss National Funds (grant to H. M., 31.065397/02) and the TransregioSonderforschungsbereich (TR-SFB11).

\section{References}

Bacconi, A., Virkki, L.V., Biber, J., Murer, H., Forster, I.C. 2005. Renouncing electroneutrality is not free of charge: Switching on electrogenicity in a $\mathrm{Na}^{+}$-coupled phosphate cotransporter. Proc. Natl. Acad. Sci. USA. 102:12606-12611

Bamberg, K., Sachs, G. 1994. Topological analysis of $\mathrm{H}^{+}, \mathrm{K}^{+}$ATPase using in vitro translation. J. Biol. Chem. 269:1690916919 
A

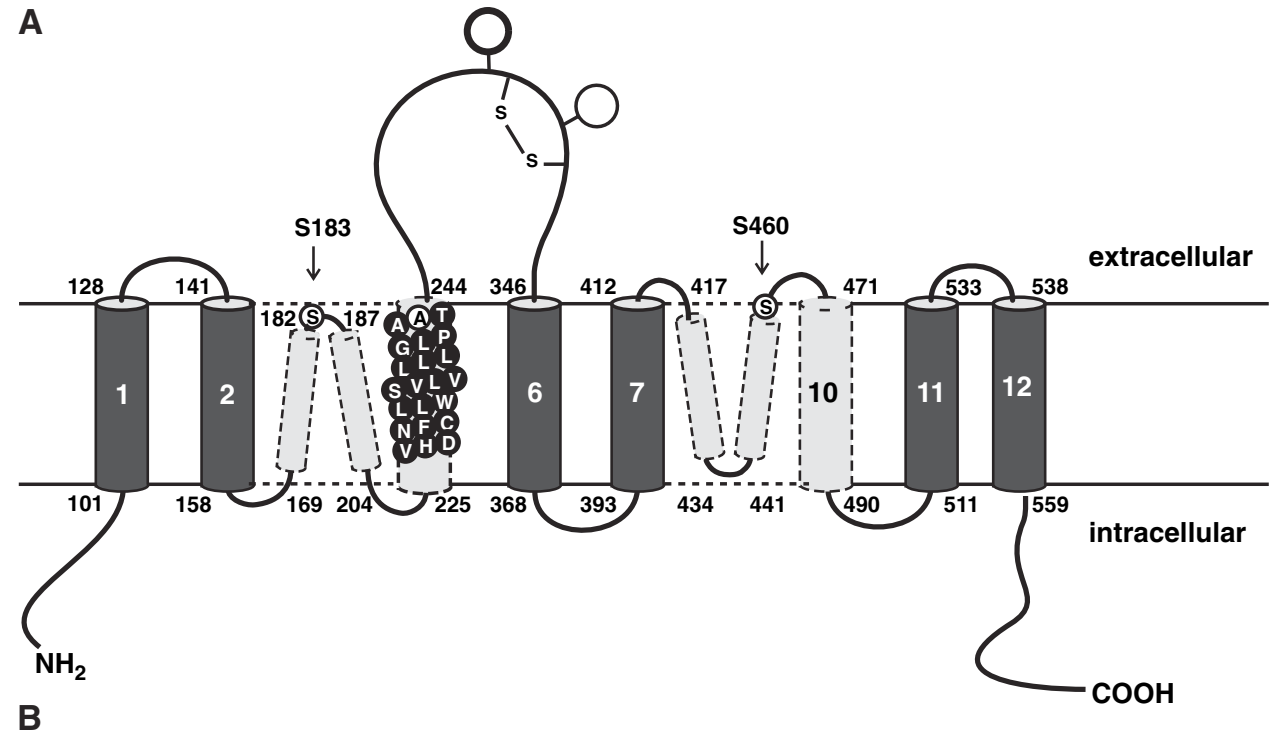

Fig. 5. (A) Model of the secondary structure of the NaPi-IIa protein. This
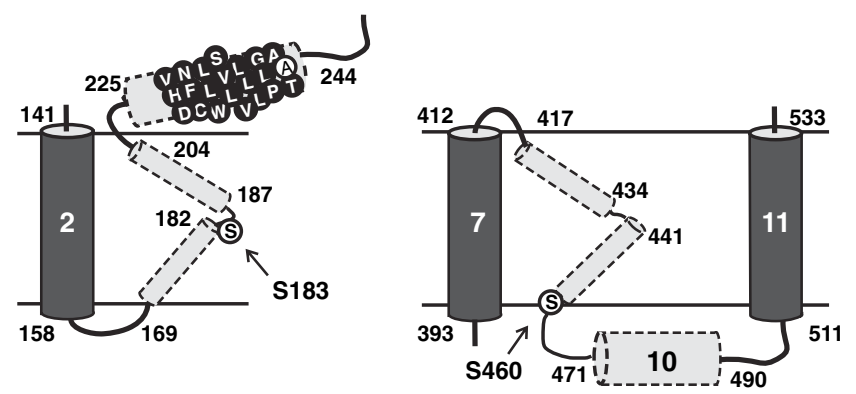
model was based on results from predictions obtained by the HMMTOP algorithm, results presented in this study and results of cysteine accessibility assays. (B) Alternative configurations of two regions comprising reentrant loops HS 3-4 and 8-9.

Bayle, D., Weeks, D., Hallen, S., Melchers, K., Bamberg, K., Sachs, G. 1997a. In vitro translation analysis of integral membrane proteins. J. Recept. Signal Transduct. Res. 17:29-56

Bayle, D., Weeks, D., Sachs, G. 1995. The membrane topology of the rat sarcoplasmic and endoplasmic reticulum calcium ATPases by in vitro translation scanning. J. Biol. Chem. 270:2567825684

Bayle, D., Weeks, D., Sachs, G. 1997b. Identification of membrane insertion sequences of the rabbit gastric cholecystokinin-A receptor by in vitro translation. J. Biol. Chem. 272:19697-19707

Custer, M., Lötscher, M., Biber, J., Murer, H., Kaissling, B. 1994. Expression of Na- $\mathrm{P}_{\mathrm{i}}$ cotransport in rat kidney: Localization by RT-PCR and immunohistochemistry. Am. J. Physiol. 266:F767-F774

Forster, I.C., Kohler, K., Biber, J., Murer, H. 2002. Forging the link between structure and function of electrogenic cotransporters: The renal type IIa $\mathrm{Na}^{+} / \mathrm{P}_{\mathrm{i}}$ cotransporter as a case study. Prog. Biophys. Mol. Biol. 80:69-108

Gerelsaikhan, T., Turner, R.J. 2000. Transmembrane topology of the secretory $\mathrm{Na}^{+}-\mathrm{K}^{+}-2 \mathrm{Cl}^{-}$cotransporter NKCC1 studied by in vitro translation. J. Biol. Chem. 275:40471-40477

Hallen, S., Branden, M., Dawson, P.A., Sachs, G. 1999. Membrane insertion scanning of the human ileal sodium/bile acid cotransporter. Biochemistry 38:11379-11388

Hallen, S., Mareninova, O., Branden, M., Sachs, G. 2002. Organization of the membrane domain of the human liver sodium/bile acid cotransporter. Biochemistry 41:7253-7266

Hartmann, E., Rapoport, T.A., Lodish, H.F. 1989. Predicting the orientation of eukaryotic membrane-spanning proteins. Proc. Natl. Acad. Sci. USA. 86:5786-5790

Hayes, G., Busch, A., Lotscher, M., Waldegger, S., Lang, F., Verrey, F., Biber, J., Murer, H. 1994. Role of N-linked glycosylation in rat renal $\mathrm{Na} / \mathrm{P}_{\mathrm{i}}$-cotransport. J. Biol. Chem. 269:24143-24149

Hirokawa, T., Boon-Chieng, S., Mitaku, S. 1998. SOSUI: Classification and secondary structure prediction system for membrane proteins. Bioinformatics. 14:378-379

Hofmann K., Stoffel W. 1993. Tm base-A database of membrane spanning protein segments. Biol. Chem. Hoppe-Seyler 374:166170

Klein, P., Kanehisa, M., DeLisi, C. 1985. The detection and classification of membrane-spanning proteins. Biochim. Biophys. Acta. 815:468-476

Kohler, K., Forster, I.C., Stange, G., Biber, J., Murer, H. 2002a. Identification of functionally important sites in the first intracellular loop of the NaPi-IIa cotransporter. Am. J. Physiol. 282:F687-F696

Kohler, K., Forster, I.C., Stange, G., Biber, J., Murer, H. 2002 b. Transport function of the renal type IIa Na $+/ \mathrm{Pi}$ cotransporter is codetermined by residues in two opposing linker regions. J. Gen. Physiol. 120:693-705

Kohler, K., Forster, I.C., Stange, G., Biber, J., Murer, H. 2003. Essential cysteine residues of the type IIa $\mathrm{Na}+/ \mathrm{Pi}$ cotransporter. Pfluegers Arch. 446:203-210

Krogh, A., Larsson, B., von Heijne, G., Sonnhammer, E.L. 2001. Predicting transmembrane protein topology with a hidden Markov model: Application to complete genomes. J. Mol. Biol. 305:567-580

Kyte, J., Doolittle, R.F. 1982. A simple method for displaying the hydropathic character of a protein. J. Mol. Biol. 157:105-132

Lambert, G., Forster, I.C., Stange, G., Biber, J., Murer, H. 1999a. Properties of the mutant Ser-460-Cys implicate this site in a functionally important region of the type $\mathrm{IIa} \mathrm{Na}+/ \mathrm{Pi}$ cotransporter protein. J. Gen. Physiol. 114:637-652 
Lambert, G., Forster, I.C., Stange, G., Kohler, K., Biber, J., Murer, H. 2001. Cysteine mutagenesis reveals novel structurefunction features within the predicted third extracellular loop of the type IIa Na ${ }^{+} / \mathrm{Pi}$ cotransporter. J. Gen. Physiol. 117: 533-546

Lambert, G., Traebert, M., Biber, J., Murer, H. 2000. Cleavage of disulfide bonds leads to inactivation and degradation of the type IIa, but not type IIb, sodium phosphate cotransporter expressed in Xenopus laevis oocytes. J. Membr. Biol. 176:143-149

Lambert, G., Traebert, M., Hernando, N., Biber, J., Murer, H. 1999b. Studies on the topology of the renal type II NaPi-cotransporter. Pfluegers Arch. 437:972-978

Mareninova, O., Shin, J.M., Vagin, O., Turdikulova, S., Hallen, S., Sachs, G. 2005. Topography of the membrane domain of the liver $\mathrm{Na}+$-dependent bile acid transporter. Biochemistry. 44:13702-13712

Melchers, K., Weitzenegger, T., Buhmann, A., Steinhilber, W., Sachs, G., Schafer, K.P. 1996. Cloning and membrane topology of a P type ATPase from Helicobacter pylori. J. Biol. Chem. 271:446-457

Monne, M., Hermansson, M., von Heijne, G. 1999. A turn propensity scale for transmembrane helices. J. Mol. Biol. 288:141145

Murer, H., Forster, I., Biber, J. 2004. The sodium phosphate cotransporter family SLC34. Pfluegers Arch. 447:763-767

Pasquier, C., Promponas, V.J., Palaios, G.A., Hamodrakas, J.S., Hamodrakas, S.J. 1999. A novel method for predicting transmembrane segments in proteins based on a statistical analysis of the SwissProt database: The PRED-TMR algorithm. Protein Eng. 12:381-385
Segawa, H., Kaneko, I., Takahashi, A., Kuwahata, M., Ito, M., Ohkido, I., Tatsumi, S., Miyamoto, K. 2002. Growth-related renal type II Na/Pi cotransporter. J. Biol. Chem. 277:1966519672

Tatishchev, S., Abuladze, N., Pushkin, A., Newman, D., Liu, W., Weeks, D., Sachs, G., Kurtz, I. 2003. Identification of membrane topography of the electrogenic sodium bicarbonate cotransporter $\mathrm{pNBC1}$ by in vitro transcription/translation. Biochemistry. 42:755-765

Tusnady, G.E., Simon, I. 2001. The HMMTOP transmembrane topology prediction server. Bioinformatics. 17:849-850

Virkki, L.V., Forster, I.C., Bacconi, A., Biber, J., Murer, H. 2005. Functionally important residues in the predicted 3rd transmembrane domain of the type IIa sodium-phosphate co-transporter (NaPi-IIa). J. Membr. Biol. 206:227-238

Virkki, L.V., Murer, H., Forster, I.C. 2006. Voltage clamp fluorometric measurements on a type II $\mathrm{Na}^{+}$-coupled $\mathrm{P}_{\mathrm{i}}$ cotransporter: Shedding light on substrate binding order. J. Gen. Physiol. 127:539-555

Virkki, L.V., Murer H., Forster I.C. 2006a. Mapping conformational changes of a type $\mathrm{IIb} \mathrm{Na}{ }^{+} / \mathrm{P}_{\mathrm{i}}$ cotransporter by voltage clamp fluorometry. J. Biol. Chem. 281:28837-28849

Werner, A., Biber, J., Forgo, J., Palacin, M., Murer, H. 1990. Expression of renal transport systems for inorganic phosphate and sulfate in Xenopus laevis oocytes. J. Biol. Chem. 265:1233112336

Zizak, M., Cavet, M.E., Bayle, D., Tse, C.M., Hallen, S., Sachs, G., Donowitz, M. 2000. $\mathrm{Na}^{+} / \mathrm{H}^{+}$exchanger NHE3 has 11 membrane spanning domains and a cleaved signal peptide: Topology analysis using in vitro transcription/translation. Biochemistry 39:8102-8112 